Comparison of hypoglycaemic, hepatoprotective and antiInflammatory activities of natural plant and callus cultures of Munroivia pinnata

By

Swarna Damayanthi Hapuarachchi

Thesis submitted to the Umiversity of Smii Jhysewnamenepura for the award of the Degree of Doctor of Philosophy in Biochemistry on 'Comparison of hypoglycaemic, hepatoprotedtive and antiinflammattomy adivitices off mattunall plkant and callus cultures of Munromiapüiinata', on $13^{\text {rd }}$ Nowember 2014. 
We certify that the candidate has incorporated all corrections, amendments and additions recommended by the examiners.

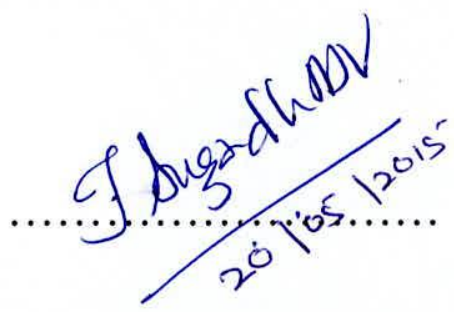

Dr. Sugandhika Suresh

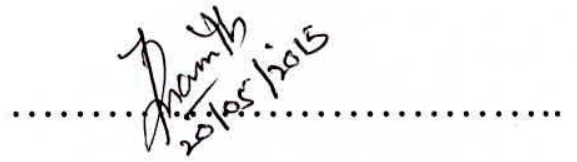

Prof. W.T.P.S.K. Senarath

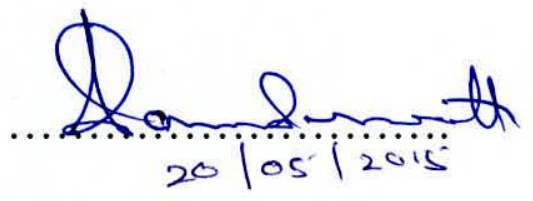

Dr. S. Handunnetti 


\section{DECLARATION BY CANDIDATE}

"The work described in this thesis was carried out by me under the supervision of Dr. Sugandhika Suresh (Department of Biochemistry, Faculty of Medical Sciences, University of Sri Jayewardenepura), Prof. W.T.P.S.K. Senarath Department of Botany, Faculty of Applied Sciences, University of Sri Jayewardenepura) and Dr. S. Handunnetti (Institute of Biochemistry, Molecular Biology and Biotchnology, University of Colombo) and a report on this has not been submitted in whole or in part to any university or any other institution for another Degree/ Diploma".

2010512015

Date

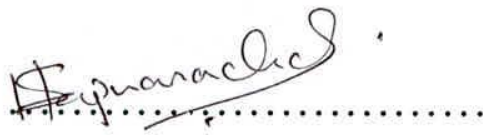

Signature of Candidate 


\section{DECLARATION BY SUPERVISORS}

"I/we certify that the above statement made by the candidate is true and that this thesis is suitable for submission to the University for the purpose of evaluation".

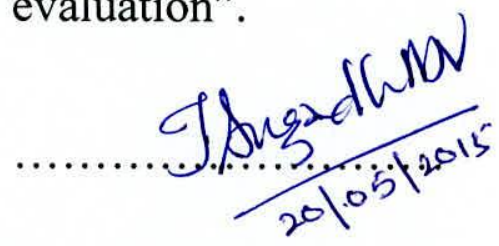

Dr. Sugandhika Suresh

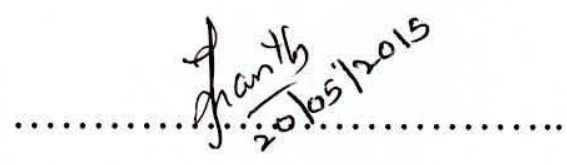

Prof. W.T.P.S.K. Senarath

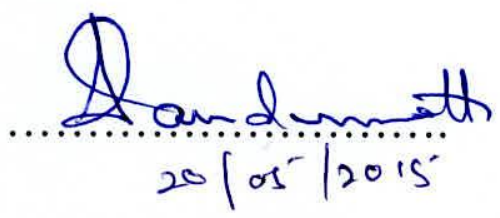

Dr. S. Handunnetti 


\section{TO MY FAMILY}




\section{TABLE OF CONTENTS}

Content

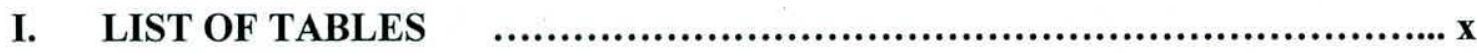

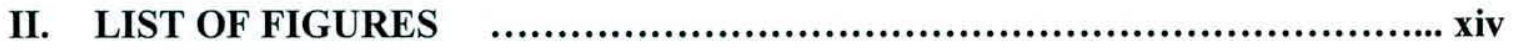

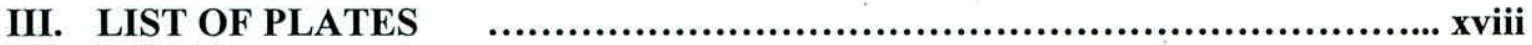

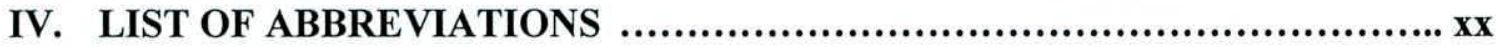

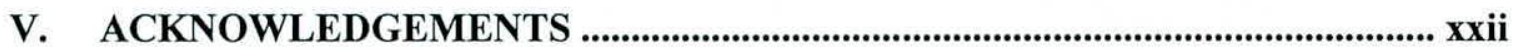

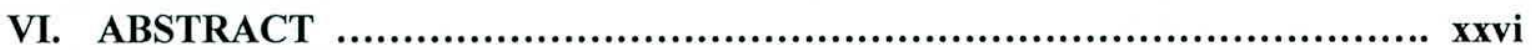

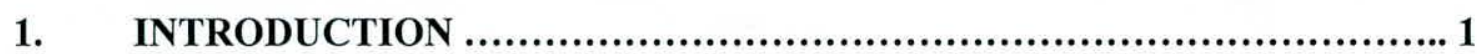

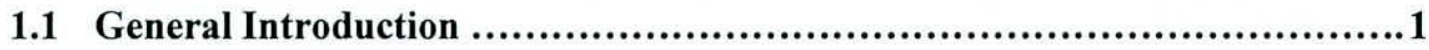

1.1.1 Importance of medicinal plants in the therapeutic systems of medicine .......... 1

1.1.2 Threats to medicinal plants and strategies for their conservation ............ 3

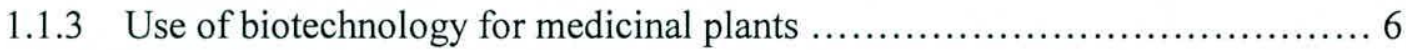

1.1.4 General introduction of Munronia pinnata .............................. 7

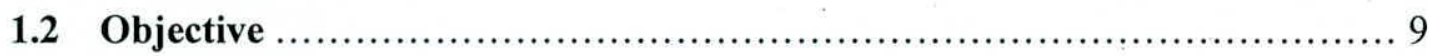

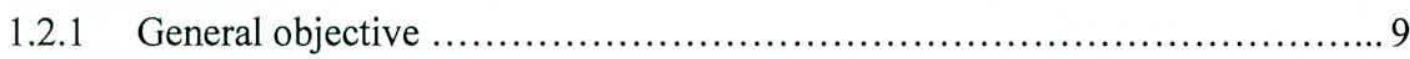

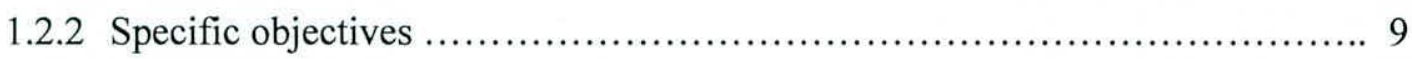

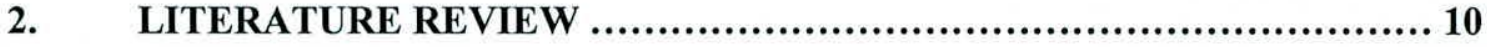

2.1 The present status of medicinal plants.................................. 10

2.1.1 Natural products as a source of establishing new drugs ...................... 11

2.2 In - vitro micropropagation of plants ...................................... 15

2.2.1 Plant cell cultures for commercial production ...........................16 
2.2.2 In-vitro callus culture ............................................... 20

2.2.3 In-vitro propagation of Munronia pinnata ............................. 21

2.3 Botanical descriptions of Munronia pinnata ................................. 22

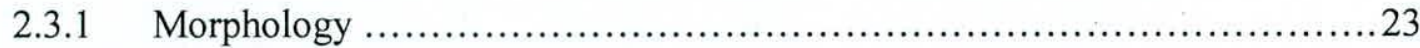

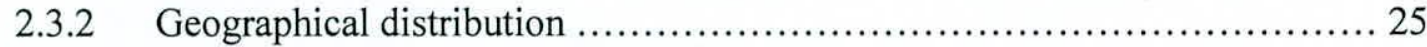

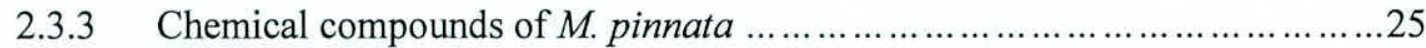

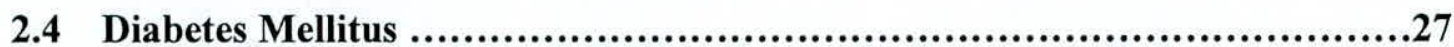

2.4.1 Diabetes in Sri Lanka................................................... 27

2. 4. 2 Hypoglycaemic herbs and their echanisms....................................... 29

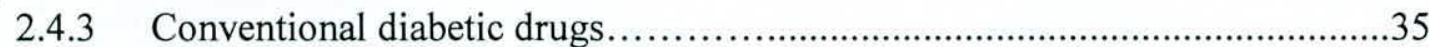

2.5 Hepatoprotective activity of medicinal plants................................. 36

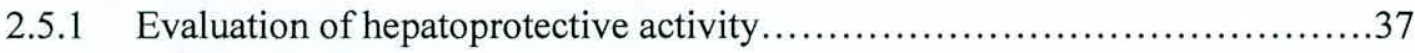

2.5.2 Mechanism of action of some selected hepatotoxins........................... 38

2.6 Anti-inflammatory activity of medicinal plants.............................40

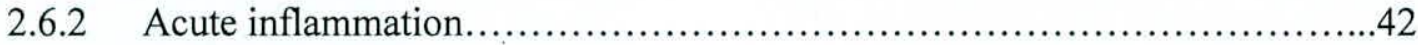

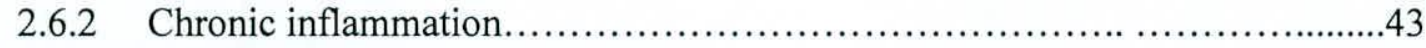

2.6.3 Experimental models of inflammation .................................44

2.6.4 Management of inflammation............................................ 45

2.7 Bioassay-guided fractionation and isolation of compounds in medicinal

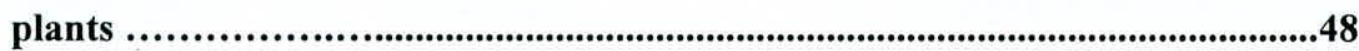

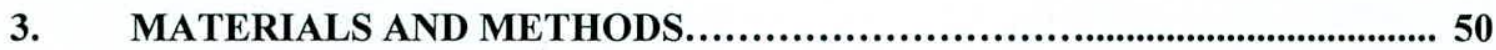

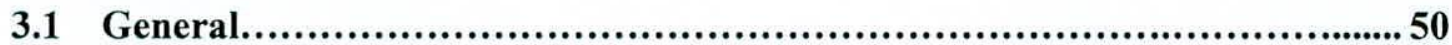




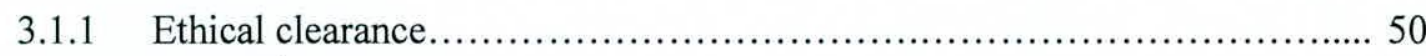

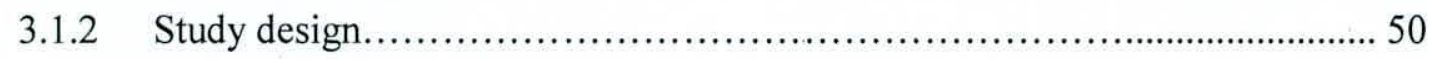

3.1.3 Oral administration of extracts and other drugs............................ 50

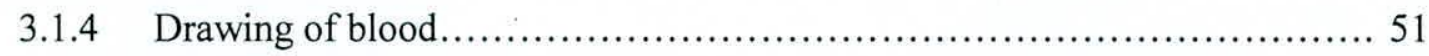

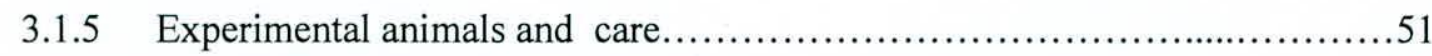

3.1.6 Collection, authentication and maintenance M. pinnata .......................53

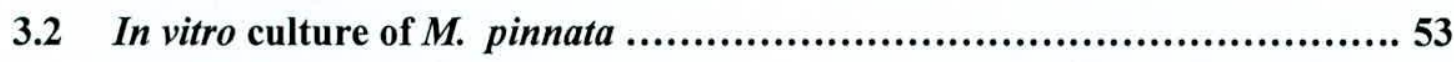

3.2.1 Determination of the best surface sterilization method for in vitro propagation

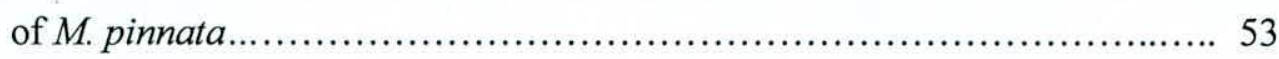

3.2.2 Determination of the best growth regulator combination and the concentrations for callus induction from leaf disc explants of M. pinnata ...................... 54

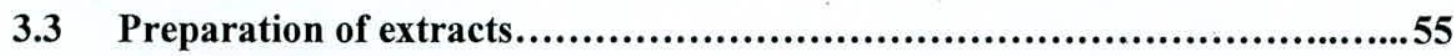

3.4 Studies on hypoglycaemic activity in Wistar rats................................ 58

3.4.1 Comparison of the two preparation methods of aqueous extracts of natural plants of M. pinnata on serum glucose concentration in normal Wistar rats. .58

3.4.2 Dose response curve and time course of the aqueous extracts (MPaq and MPCaq) of M. pinnata in healthy Wistar rats 58

3.4.3 Dose response curve and time course of the ethanol extract (MPet and MPCet) of M. pinnata in healthy Wistar rats.

3.4.4 Evaluation of the hypoglycaemic activity in healthy Wistar rats by glucose

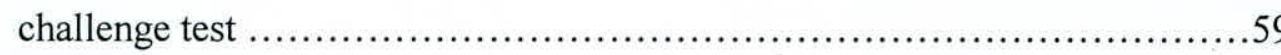

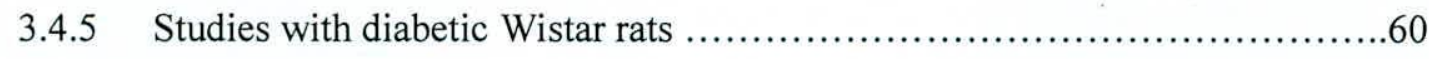




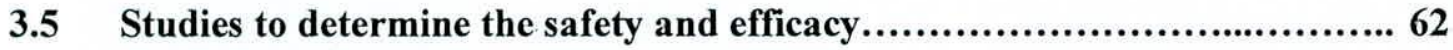

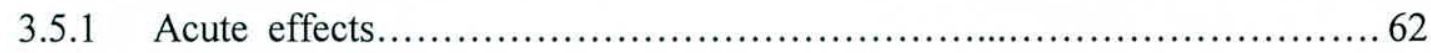

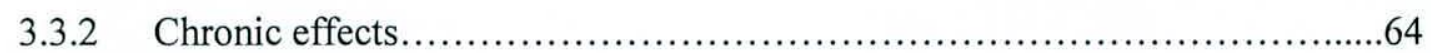

3.6 Hepatoprotective activity ...............................................66

3.7 Experiments to determine the anti- inflammatory activity.....................68

3.7.1 Anti-inflammatory activity in healthy rats.............................68

3.7.2 Anti-inflammatory activity of aqueous extracts of MPCaq and MPCet in

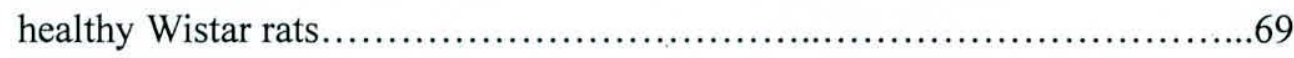

3.7.3 Anti-inflammatory activity of aqueous extracts of MPaq and MPCaq in diabetic Wistar rats................................................ 69

3.7.4 Studies on anti-inflammatory mechanisms of $M$. pinnata ......................69

3.7.4. i Effect on infiltration of rat peritoneal cells..............................69

3.7.4. ii Determination of the nitric oxide production by peritoneal cells.............71

3.7.4. iii Measurement of nitrite $\left(\mathrm{NO}_{2}{ }^{-}\right)$in culture supernatants ....................73

3.7.4. iv Assay for membrane stabilizing activity ................................73

3.7.4. v In vivo assay for anti-histamine activity ............................ 74

3.8 Clinical study on healthy and diabetic subjects............................75

3.8.1 Determination of the oral hypoglycaemic activity of natural plants and Callus cultures of $M$. pinnata in healthy volunteers.......................75

3.8.2 Determination of the oral hypoglycaemic activity of natural plants and callus cultures of M. pinnata in Type 2 diabetic patients .75 
3.8.3 Study on possible hypoglycaemic mechanisms in Type 2 diabetic patients... 77

3.8.3. i Determination of the serum insulin levels of the diabetic patients.... 77

\subsection{Extraction, characterization and isolation of bioactive compounds}

from $M$. pinnata....................................................... 78

3.9.1 Preliminary phytochemical analysis of aqueous and ethanolic extracts of M. pinnata. .78

3.9.2 Bioassay-guided fractionation and isolation of active compound/s from methanolic extract of natural plants of $M$. pinnata

3.9.2. i Extraction of natural plants of M. pinnata.

3.9.2. ii Fractionation of methanolic extract (MPm) of natural plants of $M$. pinnata....81

3.9.2. iiiFractionation of ethyl acetate extract (MPethyl ace) of natural plants of M. pinnata. .82

3.9.2. iv Fractionation of MPethyl ace-2 fraction of natural plants of $M$. pinnata .83

3.9.2. v Fractionation of MPethyl ace-2-1 fraction of natural plants of $M$. pinnata.....84

3.9.3 Extraction, isolation and identification of compounds from calli of M. pinnata.

3.9.4 Hypoglycaemic and anti-inflammatory activity of extracts and fractionations of $M$. pinnata evaluated by activity-guided methods

3.9.4. i Hypoglycaemic activity of the different extracts of natural plants and calli of M. pinnata

3.9.4. ii Hypoglycaemic activity of the fractions of methonolic extracts of natural plants and calli of $M$. pinnata .87 
3.9.4. iii Hypoglycaemic activity of the isolated compound from natural plants of

M. pinnata.

3.9.4. iv Anti-inflammatory activity of methonolic extracts of $M$. pinnata and its

fractions. 88

3.10 Statistical analysis..................................................... 89

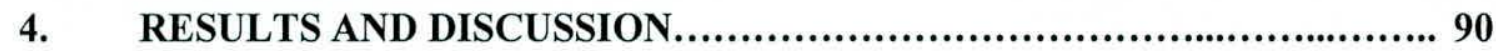

4.1 Determination of the best surface sterilization method.................... 90

4.2 Determination of the appropriate growth regulator combination and their concentrations on callus induction from leaves of $M$. pinnata............. 91

4.3 Preparation of extracts.................................................. 94

4.4 Hypoglycaemic activity in Wistar rats.............................. 94

4.4.1 Comparison of the two preparation methods of aqueous extracts of natural plants of $M$. pinnata on the serum glucose concentration in healthy

Wistar rats

4.4.2 Hypoglycaemic effect of aqueous extracts of M. pinnata (MPaq and MPCaq) on the serum glucose concentration in healthy Wistar rats. 96

4.4.3 Hypoglycaemic effect of ethanol extracts (MPet and MPCet) of M. pinnata on the serum glucose concentration in healthy Wistar rats. 100

4.4.4 Evaluation of the hypoglycaemic activity by glucose challenge test in healthy Wistar rats. 103

4.4.5 Comparison of the hypoglycaemic activity of aqueous and ethanol extracts of natural plants and calli of M. pinnata with established Ayurvedic anti diabetic 
plants in alloxan induced diabetic Wistar rats

4.4.6 Effect of aqueous extracts of natural plants and calli of M. pinnata on intestinal glucose absorption on healthy Wistar rats

\subsection{Safety effects and efficacy of long term treatment of the aqueous extracts}

of natural plants and calli of $M$. pinnata................................... 111

4.5.1 Acute effects in healthy Wistar rats.................................. 111

4.5.2 Chronic effects of the extracts of M. pinnata in healthy and diabetic

Wistar rats

4.6 Hepatoprotective activity of natural plants and calli of $M$. pinnata.......... 122

4.7 Anti-inflammatory activity of natural plants and calli of $M$. pinnata......... 128

4.7.1 Anti-inflammatory activity of aqueous and ethanolic extracts of MPaq in healthy and MPCaq Wistar rats. ...

4.7.2 Anti-inflammatory activity of MPaq and MPet extracts of M. pinnata in diabetic Wistar rats.

4.7.3 Effect of the anti-inflammatory mechanism of $M$. pinnata .................... 141

4.7.3.i Effect of M. pinnata on infiltration of rat peritoneal cells....................141

4.7.3.ii Effect of M. pinnata extracts on nitric oxide production by rat peritoneal cells.

4.7.3.iii In vitro cytotoxicity test. 146

4.7.3.iv Effect of in vitro treatment of $M$. pinnata extracts on NO production by rat peritoneal cells 146

4.7.3.v Assay for membrane stabilizing activity. 149

4.7.3.vi Determination of anti-histamine activity. 
4.8.1 Determination of the oral hypoglycaemic activity of natural plants and calli of M. pinnata in healthy volunteers

4.8.2 Determination of the oral hypoglycaemic activity of natural plants and calli of $M$. pinnata in Type 2 diabetic patients. .155

4.8.3 Possible hypglycaemic mechanisms in Type 2 diabetic patients.... 161

4.9 Studies directed at identification of active compounds .165

4.9.1 Preliminary phytochemical analysis of aqueous and ethanolic extracts of natural plants and calli of $M$. pinnata were performed using specific reagents 165

4.9.2 Extraction, fractionation of the crude plant extract and Isolation of the bioactive compounds of natural plants of $M$. pinnata.......

4.9.3 Isolation and quantification of active compound of natural plants of M. pinnata.

4.9.4 Interpretation of NMR spectral data of senecrassidiol 173

4.9.5 Fractionation of the methonolic extract of calli of $M$. pinnata 176

4.9.6 In vivo bio-assay of different extractions/ fractions of M. pinnata.

4.9.6.i Hypoglycaemic effect of the different extracts of natural plants and calli of

M. pinnata

4.9.6.ii Hypoglycaemic effect of the MPethyl ace fractions of natural plants and MPCethyl ace fractions of calli of $M$. pinnata

4.9.6.iii Hypoglycaemic effect of the isolated sesquiterpene from natural of $M$. pinnata...... 


\subsection{6.iv Anti-inflammatory activity of the isolated sesquiterpene from natural}

plants of M. pinnata

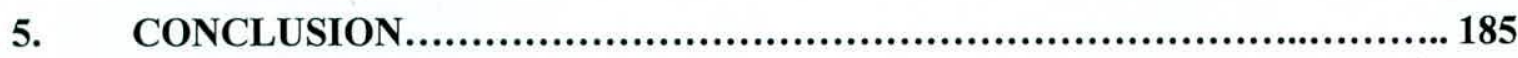

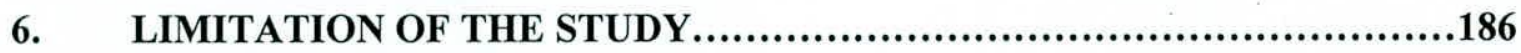

7. RECOMMENDATION FOR FUTURE STUDIES......................... 186

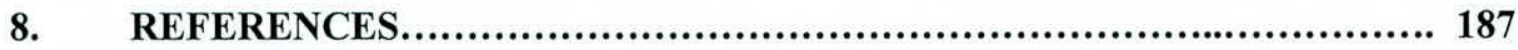

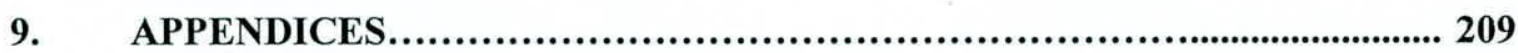

9.1 Appendix 1- Publications and communications...........................................209

9.2 Appendix 11- Constituents of MS Basal Medium.......................214

9.3 Appendix 111- Authentication of $M$. pinnata............................ 215

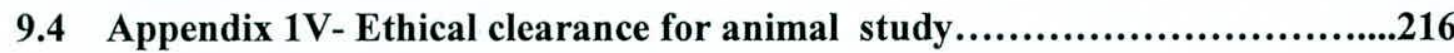

9.5 Appendix V- Ethical clearance for healthy volunteers' study $\ldots \ldots \ldots \ldots \ldots \ldots .217$

9.6 Appendix 1V- Ethical clearance for clinical study...........................218

9.10 Appendix V1- Trial Registration Confirmation Letter.......................219

9.11 Appendix V11- Diet Chart for diabetic patients..........................220

9.12 Appendix V111- Clinical Proforma....................................... 221

9.13 Appendix 1X- ${ }^{1} \mathrm{H}$ NMR and ${ }^{13} \mathrm{C}$ NMR of isolated compound................. 222 


\section{LIST OF TABLES}

Page No.

Table 1.1 Methods for germplasm conservation

Table 2.1 Some economically important plant-derived

drugs obtained commercially 14

Table 2.2 Synonyms of $M$. pinnata ......... 22

Table 2.3 NMR experiments commonly applied for structural elucidation of natural products.

Table 3.1 Different combination of Growth Regulator used in callus

induction

Table 3.2 Treatment groups of the safety study in healthy Wistar rats.

Table 3.3 Treatment groups and the selected doses of $M$. pinnata extracts

used for three months safety study in Wistar rats....

Table 3.4 Treatment groups of the hepatoprotective activity in healthy Wistar

rats 66

Table 3.5 Classes of compounds present in the aqueous and ethanolic. .78

Table.4.1 State of leaf disc explants in MS medium supplemented with $1.1 \mathrm{mg} / \mathrm{L}$

2, 4-Dand $0.2 \mathrm{mg} / \mathrm{L}$ BAP after 7 days of incubation in dark 90

Table 4.2 Mean callus diameter in MS medium supplemented with different concentrations of 2, 4-D and BAP over a period of 12 weeks.

Table 4.3 Hypoglycaemic effect of graded doses of MPaq and MPCaq in healthy

Wistar rats. 
Table 4.4 Hypoglycaemic effect of graded doses of MPet and MPCet in healthy Wistar rats

Table 4.5 Hypoglycaemic effect of graded doses of MPaq and MPCaq on serum glucose concentration of healthy rats during glucose challange 105

Table 4.6 Hypoglycaemic effect of graded doses of MPet and MPCet on serum glucose concentration of healthy rats during glucose challenge. 106

Table 4.7 Comparison of the effect of body weight and rectal temperature of healthy rats after $15^{\text {th }}$ day of the single administration of all the doses of aqueous extract of natural plants (MPaq) and calli (MPCaq) of M.pinnata.

Table 4.8 Comparison of the effect of body weight and rectal temperature of rats after $15^{\text {th }}$ day and of the single administration of all the doses of ethanol extract of natural plants and calli of $M$. pinnata....

Table 4.9 Blood /serum biochemical values of healthy rats after 14 days of the single administration of all the doses of aqueous extracts of natural plants and calli of $M$. pinnata.

Table 4.10 Blood /serum biochemical values of healthy rats after 14 days of the single administration of all the doses of ethanolic extract of natural plants and calli of M. pinnata.

Table 4.11 Comparison of the effect of body weight of healthy rats after 90 days multiple dose administration of the selecteddoses of aqueous and ethanolic extract of natural plants and calli of M.pinnata 
Table 4.12 Comparison of the effect of selected doses of aqueous and ethanolic extract of natural plants and calli of $M$. pinnata on relative organ weight $(\mathrm{g})$ per100.0 $\mathrm{g}$ body weight of healthy and diabetic rats after days 90 multiple dose administration.

Table 4.13 Biochemical serum parameters of healthy and diabetic rats after 90 days multiple dose administration of the selected doses of aqueous and ethanolic extract of natural plants and calli of M. pinnata.

Table 4.14 Comparison of the hepatoprotective effect of different extracts of

Munronia on serum marker enzymes in $\mathrm{CCl}_{4}$ induced rats

Table 4. 15 Effect of MPaq and MPCaq of M. pinnata against.

carageenan - induced paw oedema

Table 4. 16 Effect of MPet and MPCet of M. pinnata against carageenan - induced paw oedema.

Table 4. 17 Effect of MPet and MPCet of M. pinnata against carageenan - induced paw oedema in diabetic rats.

Table 4. 18 The demographic characteristics of patients in MPaq, MPcaq and control groups at base line. 156

Table 4. 19 Paraclinical characteristics of the three groups at the beginning of clinical study 156

Table 4. 20 Safety assessment of the tested decoctions. 163

Table.4. 21 Phytochemicals analysis of aqueous and ethanolic extracts of M. pinnata 166 
Table 4.22 Summary of $R_{f}$ data and colour properties of TLC spots of chloroform extracts of natural plants and calli of $M$. pinnata spraying with vanillin reagent in $\mathrm{CHCl}_{3}-\mathrm{MeOH}$ solvent system (9:1)

Table 4.23 Summary of $R_{f}$ data and colour properties of TLC spots of methanol plants and calli of $M$. pinnata spraying with vanillin reagent in EtOAc $-\mathrm{CHCl}_{3}-\mathrm{MeOH}-\mathrm{H}_{2} \mathrm{O}$ (15: 8: 4:1) solvent system. 170

Table 4. 24 Data from 2D correlation spectroscopy of compound MPethyl ace $2-1 b$

Table 4. 25 Effect of methanol extracts of $M$. pinnata and its fractions on carrageenan-induced paw oedema in Wistar rats.... 


\section{LIST OF FIGURES}

Page No.

Figure 3. 1 Procedure for nitric oxide production by peritoneal cells.... 72

Figure 3.2 Schematic representation of the extraction and bioassay of different extracts of natural plants $M$. pinnata .80

Figure 3.3 Schematic representation of the fractionation and bioassay of MPm extract of natural plants $M$. pinnata. 81

Figure 3.4 Schematic representation of the fractionation and bioassay of ethylacetae

layer (MPethyl ace) of natural plants M. pinnata.....

Figure 3.5 Schematic representation of the fractionation and bioassay of different fractions of natural plants of $M$. pinnata. 83

Figure 3.6 Schematic representation of the bioassay guided isolation of active compounds from M. pinnata..... . .84

Figure 3.7 Schematic representation of the bioassay guided isolation of of active compounds from the methonolic extract of calli of $M$. pinnata 86

Figure 4. 1 Effect of $\mathrm{MPD}_{1}$ and $\mathrm{MPD}_{2}$ on serum glucose values of healthy rats after a glucose challenge. 95 
Figure 4.2 Time cousre for different doses of MPaq (a), MPCaq (b) in healthy Wistar rats

Figure 4.3 Time course for different doses of MPet (a) and MPCet (b) in healthy Wistar rats.

Figure 4.4 Comparison of the hypoglycaemic activity of aqueous and ethanolic extract of natural plants and calli of M.pinnata with established Ayurvedic anti diabetic plants in alloxan induced diabetic Wistar rats 108

Figure 4.5 Effect of aqueous extracts of natural plants and calli of $M$. pinnata on intestinal glucose absorption in healthy Wistsr rats.

Figure 4.6 Comparison of the effect of body weight of diabetic rats after 90 days multiple dose administration of the selected doses of aqueous and ethanol extract of natural plants and calli of M. pinnata.

Figure 4.7 Comparison of the effect Munronia extracts on body weight of Wistar rats 125

Figure 4.8 Inhibition of carageenan-induced rat paw oedema following a oral administration of 11.76 and $7.84 \mathrm{~g} / \mathrm{kg}$ of MPaq and MPCaq extracts represented in charts a and $\mathrm{b}$

Figure 4.9 Inhibition of carageenan-induced rat paw oedema following a oral administration of 3.92 and $1.96 \mathrm{~g} / \mathrm{kg}$ of MPaq and MPCaq extracts represented in charts $\mathrm{c}$ and $\mathrm{d}$ 131

Figure 4.10 Inhibition of carageenan-induced rat paw oedema follow a oral administration of 1000.0 and $700.0 \mathrm{mg} / \mathrm{kg}$ of MPet and MPCet extracts represented in charts $a$ and $b$. 
Figure 4.11 Inhibition of carageenan-induced rat paw oedema following a ora

administration of 500.0 and $200.0 \mathrm{mg} / \mathrm{kg}$ of MPet and MPCet extracts

represented in charts $\mathrm{c}$ and $\mathrm{d}$. 134

Figure 4. 12 Inhibition of carageenan-induced rat paw oedema in diabetic

Wistar rats

Figure 4. 13 Effect of M. pinnata on the infiltration of rat peritoneal

cells

Figure 4.14 Standard Curve for Nitrite Concentration $\left(\mathrm{NaNO}_{2}\right)$ 144

Figure 4.15 Inhibition of in vivo NO production of rat peritoneal cells by $M$. pinnata extracts.

Figure 4.16 Inhibition of in vitro NO production of rat peritoneal cells by $M$. pinnata extracts. 148

Figure 4.17 Percent inhibition of heat - induced haemolysis of rat erythrocytes 150

Figure 4.18 Anti histamine effect of M. pinnata in Wistar rat 152

Figure 4.19.1 Oral hypoglycaemic activity of natural \& callus $M$. pinnata in healthy volunteers

Figure 4.19.2 Mean values of blood glucose levels of Type 2 diabetic patients after three months treatment of $M$. pinnata extracts 158

Figure 4.20 Glycosylated haemoglobin (HbA1c) levels after three months of M. pinnata treatment in type 2 diabetic patients. 160

Figure 4.21 Insulin concntration of after three months treatment of aqueous extracts of natural plants and calli of M. pinnata (MPaq and MPCaq) in diabetic pateints 162 


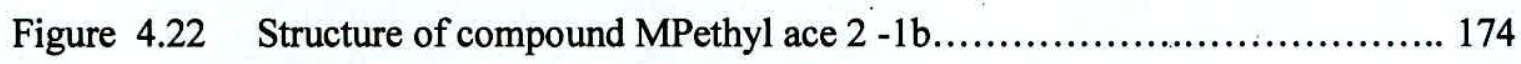

Figure 4.23 Effect of different extracts of $M$. pinnata on serum glucose

concentration of healthy Wistar rats during glucose challenge .......... 178

Figure 4.24 Hypolglycaemic effect of the different fractions of $M$. pinnata............... 180

Figure 4.25 Effect of EtacMP 2-1a of $M$. pinnata on BGL of healthy rats .............. 181 


\section{LIST OF PLATES}

Plate 2.1 a. Habit of $M$. pinnata............................................. 24

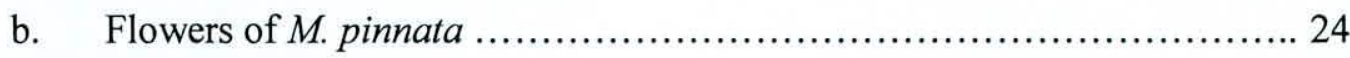

Plate $3.1 \quad$ Housing of Wistar rats............................................. 52

Plate $3.2 \quad$ Mother stocks of $M$. pinnata in the greenhouse ........................ 52

Plate $3.3 \quad$ Oral administration of extracts...................................... 52

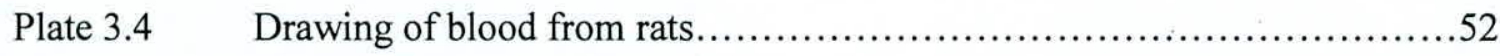

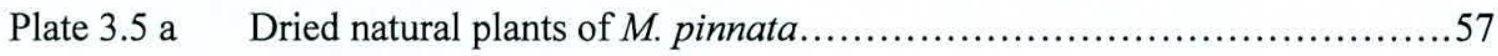

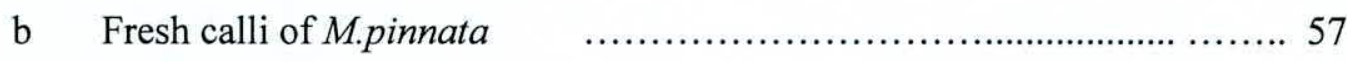

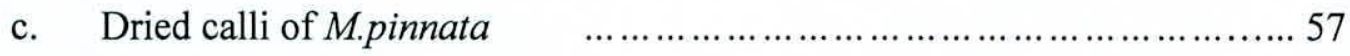

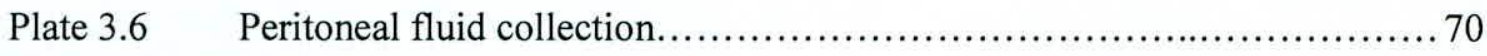

Plate 4.1 Callus cultures growing in growth regulator under aseptic condition........93

Plate 4.2 Liver microscopy using HE stained section of rat live $(x 100) \ldots \ldots \ldots \ldots \ldots 127$

Plate 4.3 Comparison of the TLC of ethanolic extracts of natural plants (MP) and calli (MPC) of $M$. pinnata in $\mathrm{CHCl}_{3}-\mathrm{MeOH}$ solvent $\quad$ system $\quad(9: 1) \ldots \ldots .168$

Plate 4.4 Comparison of the TLC of ethanolic extracts of natural plants (MP) and calli (MPc) of M. pinnata in EtOAc $-\mathrm{CHCl}_{3}-\mathrm{MeOH}-\mathrm{H}_{2} \mathrm{O}$ (15: 8: 4:1) solvent system. 168 


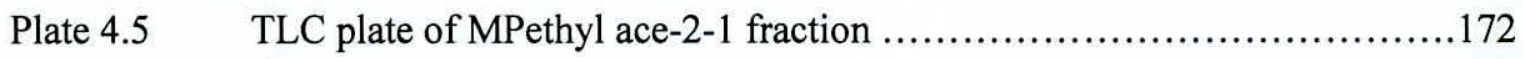

Plate 4.6 TLC of MPethyl ace 2-1b fraction $\left(\mathrm{m}_{2}\right)$ comparing with

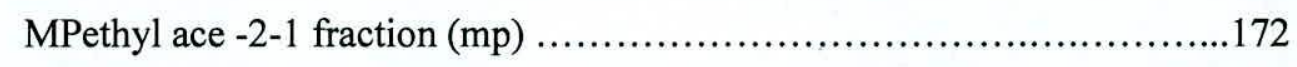

Plate 4.7 TLC plate of ethyl acetate fractions of natural plant and calli of M. pinnata. 176 


\section{LIST OF ABBREVIATIONS}

$\begin{array}{ll}2,4 \text { D } & \text { 2-4-dicloroxyphenoxyacetic acid } \\ \text { y-GT } & \text { Gamma glutamyl transferase }\end{array}$

ALT Alanine amino transferase

ALP Alkaline phosphatase

ANOVA Analysis of varience

AST Asparate amino transferase

BAP 6 - benzylaminopurine

CC column chromatography

CCM Complete Culture Medium

COX Cyclooxygenase

${ }^{13} \mathrm{C}-\mathrm{NMR} \quad$ carbon-nuclear magnetic

CRD Completely Randomized Design

ELISA Enzyme linked immunosorbant assay

HbAlc Glycated haemoglobin

${ }^{1} \mathrm{H}-\mathrm{NMR} \quad$ proton-nuclear magnetic resonance 


\begin{tabular}{|c|c|}
\hline iNOS & Inducible nitric oxide synthase \\
\hline i.p & Intraperitoneal \\
\hline LSD & Least Significance Difference \\
\hline MS & Murashige and Skoog medium \\
\hline NIDDM & Non -insulin -dependent diabetes \\
\hline NMMA & $1 \mathrm{mM}$ n-monoethyl- L- arginine acetate salt \\
\hline OD & Optical density \\
\hline OECD & Organization of Economic Co-operation Development \\
\hline OPD & Out Patient Department \\
\hline NMR & Nuclear overhauser effect spectroscopy \\
\hline NSAIDs & Non-steroidal anti-inflammatory drugs \\
\hline PBS & Posphate-Buffered Saline \\
\hline PG & Prostaglandin \\
\hline $\mathrm{RBC}$ & Red blood cell \\
\hline WHO & World health organization \\
\hline
\end{tabular}




\section{ACKNOWLEDGEMENTS}

I reverentially acknowledge my indebtedness to my supervisor, Dr. (Mrs) Sugandhika Suresh, Department of Biochemistry, Faculty of Medical sciences, University of Sri Jayewardenepura for her sharp approaches regarding the problems, which have been beyond my measures, lead me to complete this research work. She has been always kind to me in extending her valuable and important suggestions during the period of research and infused a tremendous confidence in me, by which I can able to complete this present work.

It is a pleasing privilege for me to express my cordial sense of gratefulness and regards to my other supervisors Prof. W.T.P.S.K.Senarath, Department of Botany, University of Sri Jayawardanapura and Dr, Shiromi Handunnetthi, Institute of Biochemistry, Molecular Biology and Biotechnology, University of Colombo, for their cordiality encouragement and valuable suggestions during my study period.

I express my sincere regards and profound gratefulness to Dr. Larry A. Walker, Director and Dr. Iklas Khan, Assistant Director, National Center for Natural Product Research (NCNPR), The Research Institute of Pharmaceutical Sciences, School of Pharmacy, University of Mississippi, USA for giving the opportunity to accommodate and training under experienced scientists in their groups.

I extend my deep sense of gratitude and thankfulness to Dr. Dhammika Nanayakkara, and Dr. Kumudini Senior Research Scientists in the Research Institute of Pharmaceutical Sciences, NCNPR, for giving their full support to find accommodation and advice throughout my training period. Further, I would like to express my special appreciation and thanks to Dr. Zulficar Ali, 
Senior Research Scientists in the Research Institute of Pharmaceutical Sciences, NCNPR, for encouraging my research and for allowing me to grow as a research scientist.

I am deeply indebted to Dr. Nuwan Nanayakkara and Dr. Chamu, USA; for their encouragements and kindness support to conduct the my training program at National Center for Natural Product Research (NCNPR), The Research Institute of Pharmaceutical Sciences, School of Pharmacy, University of Mississippi, USA.

I would like to acknowledge the financial support of University Grant Commission, National Center for Advanced Studies in Humanities and Higher Education Ministry, Sri Lanka, particularly in the award of research grants that provided the necessary financial support for this research work.

I express my sincere regards to Professor Sagarika Ekanayake and all other academic staff members of the Department of Biochemistry, Faculty of Medical Sciences, for their helping altitude and co-operation in carrying out my work.

I am thankful to Prof. Ajith Abeysekara and the Head of the Department of Chemistry, USJP for granting permission to carryout laboratory work during the isolation procedure of my research drug.

My thanks also due to Dr. (Mrs.) Achala Atthanayake, Additional Director, The National Herbarium, The Royal Botanical Garden, Peradeniya; for authenticating my research plant. 
I would like to express my thanks to Dr. (Mrs.) Jayasekara and Dr. Mayuri and the staff of Animal cetre, Medical Research Institute for the training on handling of animals during my research.

I am very thankful to my friends, Dr. Mallika and her family, Dr. Naohito Abe, Dr. Herath and his family and other Sri Lankan friends of the NCNPR, for their very kind support during training period at National Center for Natural Product Research (NCNPR), The Research Institute of Pharmaceutical Sciences, School of Pharmacy, University of Mississippi, USA.

I would like to extend my heartfelt gratitude to our research team mates, Mrs. Bhanuki, Mrs. Peshala, Ms. Subha, Ms. Ureshanie, and Dr. (Mrs). Saroja of the Department of Biochemistry, Faculty of Medical sciences and Mr. Priyantha of the Department of Botany, Faculty of Applied Sciences, University of Sri Jayewardenepura; for their needful suggestions and encouraging support in various aspects during my research work.

I am very thankful to my friends and colleagues, Dr. (Mrs.) Sajeeva, Dr. Kamal, Dr. Anoma, Dr.(Mrs.) Jeevani, Dr.(Mrs.) Shifa, Dr (Mrs.) Gimhani and Ms. Asha of the Department of Dravyaguna Vignana and all the staff members of the Institute of Indigenous Medicine, Rajagiriya; for their kind support during this study.

I would also like to thank all of the members of the Department of Dravyaguna Vignana, IIM; Mr. Handagama, Mr. Ashok, Mr. Kalagama and Mr. Human for their continuous help given to this work.

My thanks are due to the staff members of the Animal Centre and the Department of Biochemistry, USJP; for their numerous supports for this study. 
I must not forget to prey the name of almighty god who has blessed me with such an ideal and worship able parents Late Mr. Premasiri Hapuarechchi and late Mrs.Kumarage and my family members, for their affection, continuous encouragement and support.

I would really extend my heartful thanks to my husband, Mr. Sarath Wijewantha, for his constant inspiration and support to my work and took all at the burdens of our children's and made me free for my whole period of study.

I cannot forget the support of my beloved sons, Master Dulitha and Master Sujitha who always stayed patiently during my study period.

Of course, this research work would not have been possible without the participation of the subjects. I must not forget to extend my gratitude to the volunteers and patients who offered themselves for this study.

I really feel indebted to all those who one or other may help me in bringing up this work. I extended my thanks to them.

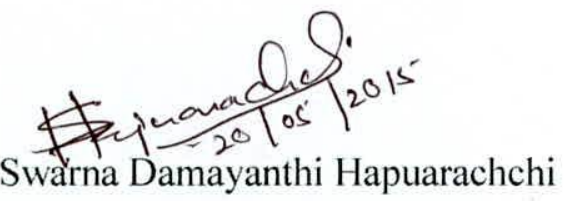




\title{
Comparison of hypoglycaemic, hepatoprotective and anti-inflammatory activities of natural plant and callus cultures of Munronia pinnata
}

\section{By Swarna Damayanthi Hapuarachchi}

\begin{abstract}
Munronia pinnata [(Wall) Theob (Meliaceae) - (Bin kohomba)] is a rare medicinal herb in Sri Lanka. Traditional physicians claim that it has been used for many diseases in the form of powder and decoction in folk medicinal practices of Sri Lanka. In-vitro propagation techniques have been applied as an attempt to meet the increasing demand for this plant. The possible use of calli as a substitute for whole plants has been querried. Despite its long record of usage in indigenous clinical practices, there is a paucity of published studies on the biological activities and active ingredients of this herb. The objective of the present study was thus to investigate the hypoglycaemic, hepatoprotective and anti-inflammatory activities of natural plants and compare such activities with the callus cultures of M. pinnata.

Leaf explants were cultured on Murashige and Skoog medium supplemented with $1.1 \mathrm{mg} / \mathrm{L} 2-4$ dicloroxyphenoxyacetic acid (2,4-D) and $0.3 \mathrm{mg} / \mathrm{L} 6$ - benzylaminopurine (BAP) to obtain callus cultures. Three leaflet type of natural plants and 3- months' old calli of M. pinnata were used for the preparation of ethanol extracts and aqueous extracts. Experimental studies with Wistar rats (healthy and diabetic) and clinical studies with diabetic patients were carried out.
\end{abstract}

A single as well as multiple doses of aqueous and ethanol extracts of the natural plants and calli significantly reduced the serum glucose concentrations in healthy and diabetic rats in a dose xxvi 
dependant manner. The optimal dose of the water extract of the natural plants was $2.0 \mathrm{~g} / \mathrm{kg}$ and it was $0.7 \mathrm{~g} / \mathrm{kg}$ for the ethanol extract. The optimal time of activity for natural plants and calli was $2 \mathrm{~h}$ after the administration of the aqueous extract and was $3 \mathrm{~h}$ for ethanol extract. Oral hypoglycaemic activity exerted by both extracts was comparable to the time course of that of standard synthetic anti diabetic drugs. Toxicity studies revealed that neither ethanol nor aqueous extracts of test samples exert any adverse effects. This is an important finding, as standard hypoglycaemic drugs, have to be administered over a relatively long period of time in the therapeutic practices and absence of adverse effects is an advantage.

Glucose challenge studies with healthy subjects and Type 2 diabetics showed a significant reduction $(23.30 \%, \mathrm{p}<0.01)$ in the serum glucose concentration levels when administered with aqueous extract of natural plants. Though the aqueous extract of calli reduced the serum glucose concentration in Type 2 diabetics, it was not as high as the aqueous extract $(6.60 \%, \mathrm{p}<0.05)$. Studies on the mechanism of action, showed a significant reduction in intestinal glucose absorption and serum glucose concentration when compared to control group (distilled water) after glucose challenge. Thus, it could be suggested that $M$. pinnata appears to exert its oral hypoglycaemic activity via several possible mechanisms. These might include the inhibition of intestinal glucose absorption, possible stimulation of insulin secretion from the beta cells of islets of Langerhans and facilitation of glucose uptake and utilization by peripheral tissues.

The hepatoprotective activity in healthy Wistar rats was investigated against $\mathrm{CCl}_{4}$ induced lipid peroxidation and hepatic injury. Results revealed that there is hepatoprotective activity against the toxic effect of $\mathrm{CCl}_{4}$, which was also supported by histological studies. Consequently, it explains that either ethanol or aqueous extracts and not only of natural plants but also of the calli 
could be used as a therapeutic regime in treatment of some hepatic disorders without any side effects.

The aqueous and ethanol extracts of both natural plants and calli possess anti-inflammatory activity in carrageenan-induced rat paw oedema model compared to the control (distilled water), in diabetic as well as healthy Wistar rats. Anti-inflammatory effect was dose-dependent and the optimal dose was $2.0 \mathrm{~g} / \mathrm{kg}$ of aqueous extract while it was $0.5 \mathrm{~g} / \mathrm{kg}$ of ethanol extract. Aqueous extracts of both natural plants and calli significantly inhibit the peritoneal phagocytic cell infiltration and impair nitric oxide production in peritoneal cells. These results indicated that anti inflammatory activity of $M$. pinnata is mediated through inhibition of NO production, phagocytic cell infiltration and anti-histamine effect. As decoction of $M$. pinnata exhibit significant anti inflammatory activity in the tested models, it may prove the scientific rationale for the use of this species in folk medicine as an anti - inflammatory agent.

During activity guided fractionation, methanol extracts of natural plants and calli were partitioned in to water and ethyl acetated TLC was done on each fraction using ethyl acetate: hexane (4:6) solvent system. The ethyl acetate extract of both natural plants and calli showed the highest reduction of serum glucose in glucose challenge studies and showed anti-inflammatory activity in healthy Wistar rats. Further fractionation of ethyl acetate extract by column chromatography with Sephadex $\mathrm{LH}_{20}$ yielded two oral hypoglycaemic fractions of natural plants and one fraction of calli. Similar $\mathrm{R}_{\mathrm{f}}$ value was obtained from one compound of the fraction 2 of ethyl acetate fraction of natural plants and calli. When compared with the TLC analysis in ethyl acetate: hexane (4:6) solvent system and these compounds appeared to contain terpenoids. Among the tested fractions, known sesquiterpene, senecrassidiol $(55.0 \mathrm{mg} ; \mathrm{w} / \mathrm{w})$ was isolated from the ethyl acetate fraction 
of methanol extract of MP and at a dose of $2.0 \mathrm{mg} / \mathrm{mL}$ exhibited significant hypoglycacmic and anti-inflammatory effects in healthy rats. The results of this study provide the scientific rationale for the use of M. pinnata in folk medicine. 